\section{Frecuencia de reingresos hospitalarios y factores asociados en afiliados a una administradora de servicios de salud en Colombia}

\author{
Hospital readmission rate and associated factors \\ among health services enrollees in Colombia
}

\author{
Frequência de readmissões e fatores associados \\ em filiados a serviços de saúde na Colômbia
}

Andrés Caballero 1

Milciades Ibañez Pinilla 2

Isabel Cristina Suárez Mendoza 1

Juan Ramón Acevedo Peña 1

\title{
Resumen
}

Los reingresos hospitalarios son frecuentes y costosos, en Colombia existe poca información. El objetivo fue establecer la frecuencia de reingresos hospitalarios por cualquier causa a 30 días y factores asociados. Se trata de un estudio analítico con cohorte retrospectiva 64.969 hospitalizaciones entre enero de 2008 y enero de 2009 en 47 ciudades colombianas 6.573 reingresos hospitalarios, prevalencia: 10,1\% (hombres 10,9\%; mujeres 9,5\%), 44,7\% en > 65 años. Mortalidad mayor en reingresos hospitalarios (5,8\% vs. 1,8\%). Mayor carga de reingresos hospitalarios en grupo sistema circulatorio. Mayor probabilidad de reingresos hospitalarios en enfermedades hematológicas y neoplasias. Estancia hospitalaria promedio durante el primer ingreso: 7 días en pacientes que reingresaron y 4,5 en quienes no. Costo total mayor para reingresos hospitalarios (USD 21.998.275): $15,8 \%$ del costo total de hospitalizaciones. Mayor prevalencia en pacientes remitidos $(18,8 \%)$ y de consulta externa $(13,7 \%)$. Los reingresos hospitalarios son frecuentes, se asocian con mayor estancia, mortalidad y costo. Mayor riesgo de reingresos hospitalarios en sexo masculino, > 65 años, pacientes remitidos de otra institución, enfermedades hematológicas y neoplasias.

Hospitalización; Readmisión del Paciente; Costos de la Atención en Salud

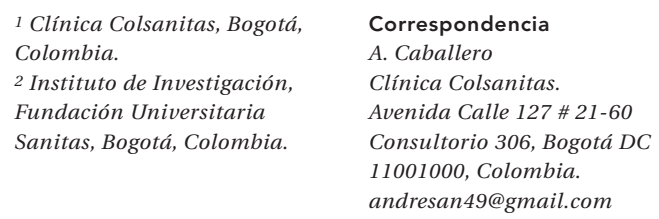

${ }^{1}$ Clínica Colsanitas, Bogotá, Colombia.

2 Instituto de Investigación, Fundación Universitaria Sanitas, Bogotá, Colombia.

\author{
Correspondencia \\ A. Caballero \\ Clínica Colsanitas. \\ Avenida Calle 127 \# 21-60 \\ Consultorio 306, Bogotá DC \\ 11001000, Colombia. \\ andresan49@gmail.com
}




\section{Introducción}

Los reingresos hospitalarios son eventos frecuentes, potencialmente prevenibles, costosos, y asociados a alta morbi-mortalidad 1,2. Pueden definirse como admisiones a un hospital dentro de cierto periodo después de haber egresado del mismo u otro centro hospitalario 2,3,4; el tiempo definido para su medición varía y la tendencia es utilizar como referencia los primeros 30 días posteriores al egreso ${ }^{5}$. La tasa de reingresos en este lapso para diferentes centros médicos y países oscila entre $5 \%$ y $19.6 \% 2,3,6$.

Se han descrito varios factores asociados al reingreso: estancia hospitalaria, severidad de la enfermedad, comorbilidades, número de ingresos previos a urgencias $u$ hospitalización, sexo masculino, ser mayor de 65 años y deficiencias en el cuidado 7,8,9; la baja calidad de vida, los síntomas depresivos y el bajo nivel socioeconómico también se han asociado con reingresos 10,11,12. Varias enfermedades y grupos de diagnóstico se han asociado a reingresos 2,3, $13,14,15,16,17,18,19,20,21,22,23$. De manera contraria, algunos estudios han descrito factores asociados con menor riesgo de reingresos tales como la intervención quirúrgica y el parto 24 .

En nuestro país son escasas las publicaciones de reingresos y el impacto que generan sobre los recursos y la prestación de los servicios de salud. El objetivo de este estudio es establecer las características del reingreso hospitalario a 30 días, los factores y costos asociados, al igual que los grupos de diagnóstico identificados con mayor frecuencia, en una cohorte de pacientes no obstétricos afiliados a un grupo de compañías promotoras y administradoras de planes de salud en Colombia.

\section{Métodos}

Estudio analítico de cohorte retrospectiva de egresos hospitalarios. Se incluyeron pacientes afiliados a la Organización Sanitas Internacional (grupo de compañías promotoras y administradoras de planes de salud) y hospitalizados en clínicas y hospitales privados que aportan información y reciben auditoría de la Organización en 47 ciudades de Colombia). Fueron excluidos del análisis los casos que no contaron con el tiempo de observación de 30 días después del egreso y aquellos con admisión inicial relacionada con atención obstétrica. La variable de desenlace principal fue el reingreso por cualquier causa dentro de los primeros 30 días posteriores al egreso hospitalario, incluyendo pacientes que hayan ingresado y reingresado en más de una ocasión.
Otras variables de desenlace fueron la estimación de costos globales desde la perspectiva del asegurador y estado de egreso (vivo o muerto). Las variables pronosticas fueron: grupos de diagnóstico de egreso (Clasificación Internacional de las Enfermedades, 10ª revisión - CIE-10), origen del ingreso (urgencia, programado, trasladado de otra institución, consulta externa), tipo de egreso (médico, quirúrgico), estancia hospitalaria, edad y sexo.

Se incluyeron ingresos y reingresos identificados entre el 1o enero de 2008 y el 31 de enero de 2009 en 47 ciudades colombianas. Esto nos permite analizar un año completo de hospitalizaciones (de 1o de enero a 31 de diciembre de 2008) y evaluar en un año completo los reingresos a 30 días (hasta 31 de enero 2009). La Organización Sanitas Internacional en la época del estudio, contaba con 1.072.863 afiliados de los cuales $27,5 \%$ pertenecen a estratos socioeconómicos altos (estratos 4-6, 295.742 afiliados), que además de tener la cobertura básica de salud, poseen planes complementarios de salud. El resto $(72,5 \%)$ pertenece a los estratos 1 a 3, que sólo tienen el plan obligatorio o básico en cobertura de salud. El estudio toma como universo todos los afiliados a la Organización que tenían derecho a usar sus servicios (1.072.863).

La recolección y registro de la información fue efectuada por profesionales de la salud y auditores de la Organización. La sistematización de la información y el registro se hizo en hojas de cálculo de Excel (Microsoft Corp., Estados Unidos). Para la depuración y procesamiento de datos se utilizó el paquete estadístico SPSS versión 17.0 (SPSS Inc., Chicago, Estados Unidos).

La calidad de la información sistematizada se verificó con distribuciones de variables y cruces lógicos entre éstas, donde se corrigieron los errores detectados; en caso de no ser posible su corrección, se eliminó el dato. Los sesgos de selección se controlaron tomando en forma consecutiva la totalidad de egresos hospitalarios para el periodo de estudio. La pérdida de datos fue menor al $2 \%$ sin ningún tipo de patrón que generara sesgo. Los sesgos de clasificación relacionados con la información se controlaron mediante la eliminación de datos inconsistentes, los cuales no se pudieron verificar por ninguna fuente de recolección, fechas de ingreso, egreso y reingreso $\mathrm{y}$ en las variables independientes, género, diagnóstico y estancias.

\section{Análisis estadístico}

La descripción de variables cualitativas en los factores demográficos y clínicos (sexo, grupos de edad, diagnósticos, estado al egreso y 
mortalidad) se realizó mediante distribuciones de frecuencias absolutas y relativas expresadas en porcentajes; el reingreso hospitalario a los 30 días se midió como una proporción expresada en porcentaje. En las variables cuantitativas (edad, estancia y costo global) se utilizaron medidas de tendencia central (promedio y mediana) y medidas de dispersión (rango y desviación estándar). Con el análisis bivariante se evaluó la asociación de variables cualitativas demográficas y clínicas con el reingreso hospitalario, mediante la prueba asintótica de asociación ji-cuadrado con corrección por continuidad de Yates o el test de razón de verosimilitud. En las variables cuantitativas que cumplieron el supuesto de normalidad (estancia hospitalaria y costo global) con reingreso hospitalario, se utilizó la prueba T-Student o análisis de varianza (ANOVA, más de dos grupos) para muestras independientes; se evaluó previamente la normalidad con el test de KolmogorovSmirnov y Shapiro Wilk; la homogeneidad de varianzas con el Test de Levene. En caso de no cumplirse los supuestos, se utilizó la prueba noparamétrica asintótica de Mann Whitney o ANOVA no-paramétrico de Kruskall-Wallis.

El conjunto de variables explicativas de reingreso hospitalario a los 30 días se obtuvo mediante un modelo de regresión logística incondicional. Debido al diseño de cohorte retrospectiva, en su direccionalidad y tiempo completos de observación del reingreso a los 30 días, se tomó el odds ratio (OR) como estimador del riesgo relativo (RR). La bondad de ajuste del modelo se midió con la prueba de Hosmer-Lemeshow y la prueba de verosimilitud. Las pruebas estadísticas se evaluaron a un nivel de significancia de $\mathrm{p}<0.05$.

\section{Resultados}

La cohorte total estuvo conformada por 64.969 hospitalizaciones, lo que indica una tasa de hospitalización de 8,3\%, excluyendo las causas relacionadas con el embarazo, en el año de análisis. Se encontró mayor número de mujeres, el rango de edad varió entre menos de un año de vida (neonatos) y 108 años, el promedio de edad fue 44,8 años (D.E $\pm 28,7$ ) con mediana de 50 años (Tabla 1). El origen de hospitalización fue: urgencias 49.048 (75,5\%), programados 11.068 (17\%), trasladados de otra institución $4.048(6,2 \%)$ y consulta externa 805 (1,2\%). Las especialidades con mayor hospitalización fueron medicina interna $20,1 \%$, pediatría $16,8 \%$, cirugía general $15,4 \%$ y cardiología $8,1 \%$.

Se presentaron 6.573 reingresos durante el periodo de estudio. La prevalencia de reingreso por cualquier causa fue $10,1 \%$, la cual fue ma- yor en los hombres $(10,9 \%$ vs. $9,5 \%$; $\mathrm{p}<0.001) \mathrm{y}$ el $44,7 \%$ de los reingresos ocurrió en personas mayores de 65 años de edad. En el análisis de la variable por grupos de edad se encontraron diferencias significativas $\mathrm{p}<0.001$ (Tabla 2 ).

La probabilidad de reingreso hospitalario por diagnóstico de egreso fue mayor en el grupo de enfermedades hematológicas $(22,4 \%)$, el cual aportó 1,4\% de los reingresos. El grupo de diagnóstico con mayor número absoluto de reingresos fue el del sistema circulatorio, con participación de 19,5\% (Tabla 3). Cinco grupos de diagnóstico aportaron el 66,3\% de los reingresos: enfermedades del sistema circulatorio, enfermedades del sistema respiratorio, neoplasias, enfermedades del sistema digestivo, y enfermedades del sistema genitourinario.

Se encontraron diferencias estadísticamente significativas en la prevalencia de reingreso por origen de hospitalización: traslado de otra institución $(18,8 \%)$, consulta externa $(13,7 \%)$, urgencias $(10,3 \%)$ y programados $(5,9 \%)(\mathrm{p}<0,001)$; sin embargo, el número absoluto mayor de reingresos $(n=5.052)$, correspondió a hospitalización por urgencias. De los reingresos en pacientes que habían ingresado programados, el 46,6\% $(n=305)$ correspondieron al grupo de diagnóstico de neoplasias.

\section{Análisis multivariado}

En el modelo final para explicar el reingreso se incluyeron las siguientes variables: grupos etario, sexo, grupo de diagnóstico de egreso y origen de hospitalización. Comparando con el grupo entre 5 y 14 años, el grupo con mayor riesgo significativo de reingreso fue el de 65 años y más, con un RR $=2,96$. Los hombres presentaron mayor riesgo de reingresos $(\mathrm{RR}=1,19)$. Los grupos de diagnóstico de egreso asociados significativamente con reingreso fueron enfermedades hematológicas de los órganos hematopoyéticos ( $R R=4,31)$, neoplasias $(\mathrm{RR}=2,34)$, enfermedades del sistema nervioso $(\mathrm{RR}=2,27)$ y trastornos mentales y del comportamiento ( $R R=2,08)$. Al comparar con los ingresos programados, los ingresos de otro origen se relacionaron con mayor riesgo de reingresos (traslado de otra institución $\mathrm{RR}=4,60$; consulta externa $\mathrm{RR}=2,95$ y urgencias $\mathrm{RR}=2,46$ (Tabla 4 ).

\section{Estancia hospitalaria}

La primera estancia hospitalaria de los pacientes que reingresaron $(7,07 \pm 8,45$ días, mediana $=5,0)$ fue significativamente mayor que la detectada en quienes no reingresaron $(4,48 \pm 6,61$ días; mediana $=3,0 ; p<0,001)$. 
Tabla 1

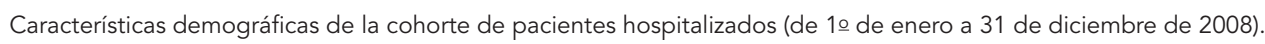

\begin{tabular}{lcc}
\hline Variable & Hospitalizaciones & $\%$ \\
& $\mathbf{n}$ & \\
\hline Sexo & & 44,2 \\
$\quad$ Hombre & 28.719 & 55,8 \\
Mujer & 36.250 & \\
Edad (años) & & 8,7 \\
$<1$ & 5.670 & 8,4 \\
$1-4$ & 5.425 & 6,4 \\
$5-14$ & 4.168 & 20,4 \\
$15-44$ & 13.244 & 23,5 \\
$45-64$ & 15.265 & 32,6 \\
$\geq 65$ & 21.197 & 100,0 \\
Total & 64.969 & \\
\hline
\end{tabular}

Nota: total de la población asegurada $=1.072 .863$ personas; total de personas hospitalizadas en un año $=64.969$.

Tabla 2

Prevalencia de reingreso a $<30$ días del egreso según grupos de edad.

\begin{tabular}{cccc}
\hline Variable & Egresos & \multicolumn{2}{c}{ Reingresos * } \\
& $\mathbf{n}$ & $\mathbf{n}$ & $\%$ \\
\hline Edad (años) & & & \\
$<1$ & 5.670 & 519 & 9,2 \\
$1-4$ & 5.425 & 289 & 5,3 \\
$5-14$ & 4.168 & 210 & 5,0 \\
$15-44$ & 13.244 & 1.045 & 7,9 \\
$45-64$ & 15.265 & 1.575 & 10,3 \\
$\geq 65$ & 21.197 & 2.935 & 13,8 \\
Total & 64.969 & 6.573 & 10,1 \\
\hline
\end{tabular}

* $p<0,001 ; \chi^{2}$ asintótica con corrección.

\section{Costos}

La mediana del costo fue significativamente mayor en los pacientes que reingresaron, que en los que no reingresaron $(p<0,001)$. Los reingresos correspondieron a $15,9 \%$ del costo global de hospitalizaciones (Tabla 5). No tenemos datos de los costos por grupos de diagnóstico.

\section{Mortalidad}

La proporción de mortalidad intrahospitalaria en la cohorte general fue de $2,2 \%(n=1.426)$. La mortalidad en los reingresos fue significativa- mente mayor que la de aquellos que no reingresaron $(5,8 \%$ vs. $1,8 \%$; $\mathrm{p}<0,001)$.

\section{Discusión}

Este estudio describe frecuencia, costos, mortalidad y establece factores asociados a reingresos por cualquier causa en una gran cohorte de pacientes afiliados a un grupo de compañías promotoras y administradoras de planes de salud en hospitales y clínicas de 47 ciudades colombianas. 
Probabilidad de reingreso y participación por capítulos de diagnóstico de la Clasificación Internacional de las Enfermedades, 10ạ revisión (CIE-10).

\begin{tabular}{|c|c|c|c|c|}
\hline Capítulo CIE-10 & $\begin{array}{l}\text { Número de } \\
\text { egresos por } \\
\text { capítulo }\end{array}$ & $\begin{array}{c}\text { Probabilidad de } \\
\text { reingreso (\%) }\end{array}$ & $\begin{array}{l}\text { Número de } \\
\text { reingresos }\end{array}$ & $\begin{array}{l}\text { Participación del } \\
\text { capítulo en los } \\
\text { reingresos (\%) }\end{array}$ \\
\hline Enfermedades del sistema circulatorio & 9.168 & 14,0 & 1.284 & 19,5 \\
\hline Enfermedades del sistema respiratorio & 10.555 & 9,7 & 1.029 & 15,7 \\
\hline Neoplasias & 5.478 & 13,6 & 745 & 11,3 \\
\hline Enfermedades del sistema digestivo & 9.578 & 7,0 & 669 & 10,2 \\
\hline Enfermedades del sistema genitourinario & 6.972 & 9,1 & 634 & 9,6 \\
\hline Ciertas enfermedades infecciosas y parasitarias & 4.611 & 8,5 & 390 & 5,9 \\
\hline $\begin{array}{l}\text { Traumatismos, envenenamientos y ciertas patologías de } \\
\text { causa externa }\end{array}$ & 2.510 & 12,0 & 302 & 4,6 \\
\hline Síntomas y signos no explicados por otra enfermedad & 2.525 & 10,6 & 267 & 4,1 \\
\hline Enfermedades del sistema nervioso & 1.882 & 13,0 & 244 & 3,7 \\
\hline Trastornos mentales y del comportamiento & 1.941 & 11,7 & 227 & 3,5 \\
\hline Enfermedades endocrinas nutricionales y metabólicas & 1.824 & 9,4 & 172 & 2,6 \\
\hline $\begin{array}{l}\text { Enfermedades del sistema osteomuscular y tejido } \\
\text { conectivo }\end{array}$ & 2.746 & 5,8 & 159 & 2,4 \\
\hline Afecciones del periodo perinatal & 2.153 & 6,8 & 146 & 2,2 \\
\hline Enfermedades de piel y tejido celular subcutáneo & 1.410 & 9,1 & 128 & 1,9 \\
\hline $\begin{array}{l}\text { Enfermedades de sangre, órganos hematopoyéticos, } \\
\text { sistema inmunológico }\end{array}$ & 425 & 22,4 & 95 & 1,4 \\
\hline Malformaciones congénitas y cromosómicas & 396 & 8,8 & 35 & 0,5 \\
\hline Factores que influyen en el estado de salud & 298 & 7,7 & 23 & 0,3 \\
\hline Enfermedades del oído y apófisis mastoides & 394 & 5,6 & 22 & 0,3 \\
\hline Enfermedades de ojo y anexos & 105 & 1,9 & 2 & 0,0 \\
\hline Total & 64.969 & 10,1 & 6.573 & 100,0 \\
\hline
\end{tabular}

\section{Frecuencia}

La tasa de reingresos fue $10,1 \%$ a 30 días. Esta cifra es similar a la reportada por van Walraven et al. 7 y Zapatero et al. ${ }^{25}$, quienes describieron una frecuencia de reingresos de $8 \%$ y $12,4 \%$, y menor que las reportadas por Hasan et al. 26 y Jencks et al. 2, 17,5\% y 19,6\% respectivamente. En general la frecuencia de los reingresos hospitalarios a los 30 días presenta grandes variaciones entre hospitales y regiones; en estudios de varias regiones de Estados Unidos y Canadá, la tasa osciló entre $7,5 \%$ y $23,2 \% 2,7$. Las razones que explican estas diferencias son amplias, dado que los reingresos hospitalarios obedecen a relaciones complejas entre el estado de salud y funcionalidad, los cuidados y manejo intrahospitalario, interacciones entre el paciente, su entorno familiar y la comunidad 27 . Estas variaciones se observan aún entre varios centros para pacientes con una misma patología, como se puede observar en los hallazgos de Epstein et al. ${ }^{28}$. La inclusión en nuestro estudio de pacientes de todos los grupos de edad y de diversas especialidades, características de nuestro sistema de salud y aseguramiento de los pacientes, la recolección de la información en diferentes regiones del país, la existencia de programas de seguimiento en algunas zonas son factores que, entre muchos otros, pueden explicar las diferencias encontradas en el presente estudio con otros trabajos; sin embargo, podemos señalar que nuestros hallazgos se encuentran dentro del rango descrito en la literatura.

\section{Grupos de edad}

En nuestra cohorte la prevalencia de reingresos según la edad, presentó un comportamiento en forma de J, que corresponde a mayor reingreso en los extremos de edad, sobre todo en 65 años y más. Se ha descrito mayor frecuencia de reingresos en personas de edad avanzada 2,25,26,29, y en el primer año de vida 30,31. La mayor probabilidad de reingresos en el grupo de edad mayor 
Modelo de regresión logística para estimación de riesgo de reingreso según factores asociados.

\begin{tabular}{|c|c|c|c|}
\hline Variable & RR estimado & IC95\% & Valor de $p$ * \\
\hline \multicolumn{4}{|l|}{ Grupos etarios (años) } \\
\hline$<1$ & 2,11 & $1,77-2,52$ & $<0,01$ \\
\hline $1-4$ & 1,04 & $0,87-1,25$ & 0,66 \\
\hline $5-14$ & 1,00 & & \\
\hline $15-44$ & 1,77 & $1,52-2,08$ & $<0,01$ \\
\hline $45-64$ & 2,35 & $2,02-2,74$ & $<0,01$ \\
\hline$\geq 65$ & 3,02 & $2,61-3,50$ & $<0,01$ \\
\hline \multicolumn{4}{|l|}{ Sexo } \\
\hline Hombre & 1,17 & $1,11-1,24$ & $<0,01$ \\
\hline Mujer & 1,00 & & \\
\hline \multicolumn{4}{|l|}{ Grupo diagnóstico de egreso } \\
\hline Enfermedades de sangre, órganos hematopoyéticos, sistema inmunológico & 3,30 & $2,48-4,38$ & $<0,01$ \\
\hline Neoplasias & 2,89 & $2,41-3,46$ & $<0,01$ \\
\hline Malformaciones congénitas y cromosómicas & 1,77 & $1,19-2,62$ & $<0,01$ \\
\hline Enfermedades del sistema nervioso & 1,76 & $1,43-2,18$ & $<0,01$ \\
\hline Traumatismos, envenenamientos y ciertas patologías de causa externa & 1,53 & $1,25-1,88$ & $<0,01$ \\
\hline Trastornos mentales y del comportamiento & 1,50 & $1,21-1,86$ & $<0,01$ \\
\hline Enfermedades del sistema genitourinario & 1,40 & $1,17-1,68$ & $<0,01$ \\
\hline Síntomas y signos no explicados por otra enfermedad & 1,39 & $1,13-1,71$ & $<0,01$ \\
\hline Enfermedades endocrinas nutricionales y metabólicas & 1,36 & $1,08-1,71$ & $<0,01$ \\
\hline Factores que influyen en el estado de salud & 1,27 & $0,80-2,01$ & 0,31 \\
\hline Enfermedadesde piel y tejido celular subcutáneo & 1,22 & $0,95-1,56$ & 0,12 \\
\hline Afecciones del periodo perinatal & 0,87 & $0,67-1,13$ & 0,28 \\
\hline Enfermedades del oído y apófisis mastoides & 0,78 & $0,49-1,23$ & 0,28 \\
\hline Enfermedades de ojo y anexos & 0,29 & $0,07-1,21$ & 0,09 \\
\hline Enfermedades del sistema osteomuscular y tejido conectivo & 1,00 & & \\
\hline \multicolumn{4}{|l|}{ Origen de la hospitalización } \\
\hline Traslado de otra institución & 4,60 & $4,07-5,20$ & $<0,01$ \\
\hline Consulta externa & 2,95 & $2,36-3,68$ & $<0,01$ \\
\hline Urgencias & 2,46 & $2,24-2,71$ & $<0,01$ \\
\hline Programado & 1,00 & & \\
\hline
\end{tabular}

IC95\%: intervalo del 95\% de confianza; RR: riesgo relativo.

* Se considera estadísticamente significativo valores de $p<0,05$.

de 65 años puede relacionarse con la presencia más frecuente en ellos de varias comorbilidades, marcadores de fragilidad o discapacidad severa 32,33 , aspectos que no evaluamos.

\section{Sexo}

Encontramos mayor frecuencia de reingreso en hombres (10,9\% vs. 9,5\%). En varios trabajos se ha señalado un mayor número de hombres readmitidos, tanto en estudios generales de reingresos 2,28 como en trabajos de patologías específicas, tales como neumonía, enfermedad pulmonar obstructiva crónica y fallo cardíaco 12,24,34,35, pero en otros estudios el sexo no parece relacionarse con el reingreso ${ }^{32}$. Se requieren estudios adicionales que permitan clarificar el papel del sexo como factor de riesgo para reingreso, y los posibles factores relacionados.

\section{Grupo de diagnóstico}

Cinco capítulos de la CIE-10 se asociaron a cerca de dos tercios de los ingresos (datos no mostrados, se puede inferir de la Tabla 3) y la misma proporción de los reingresos: enfermedades del sistema circulatorio, enfermedades del sistema respiratorio, neoplasias, enfermedades del 
Costo hospitalario global y mediana por paciente de reingresos a 30 días y hospitalizaciones no reingresos.

\begin{tabular}{lccc}
\hline Variable & $\mathbf{n}$ & Mediana (costo por paciente) & Costo total (USD) * \\
& & & \\
Reingreso & & 1.411 & 21.998 .275 \\
Sí & 6.529 & 959 & 116.603 .963 \\
No & 57.919 & - & - \\
Sin dato & 521 & & 138.602 .238 \\
Total & 64.969 & & \\
\hline
\end{tabular}

* Valores en 2008.

sistema digestivo y enfermedades del sistema genitourinario. Las enfermedades del sistema circulatorio y las neoplasias tuvieron, además, una tasa de reingresos a 30 días mayor a $13 \%$, y las enfermedades de la sangre mayor de $20 \%$. Entre más de 11 millones de hospitalizaciones de Medicare, las tasas más altas de reingresos ocurrieron después de egresos por insuficiencia cardiaca, neumonía y enfermedad pulmonar obstructiva crónica ${ }^{2}$. En el estudio español 25 de casi un millón de egresos de Medicina Interna, esas mismas categorías de diagnóstico y otras ocho que incluyen el cáncer se asociaron con el riesgo de reingreso. Esos estudios usaron métodos basados en grupos relacionados por diagnóstico, y no capítulos de CIE-10 como el nuestro, sin embargo, nuestro estudio concuerda con ellos en el predominio de las enfermedades cardiovasculares, pulmonares y neoplásicas en los pacientes que reingresan. Las diferencias en la conformación de las cohortes, las diferentes herramientas y clasificaciones utilizadas para la medición de los reingresos y las particularidades de cada sistema de salud pueden explicar las diferencias encontradas con esos estudios.

\section{Origen del ingreso}

Encontramos que el ingreso programado se asociaba muy claramente a menor riesgo de reingreso que el ingreso por urgencias, o por traslado de otra institución, o directamente de consulta externa. El reingreso en pacientes que provienen de consulta externa puede relacionarse con que en este grupo se incluyen sujetos con enfermedades hemato-oncológicas, inmunológicas y neoplásicas y que se admiten de manera repetida desde consulta para procedimientos terapéuticos periódicos. La posible explicación del menor riesgo de reingreso con las hospitalizaciones programadas es que la mayoría de ellas corresponden a procedimientos quirúrgicos no urgentes, en los cuales son más controladas o predecibles las consecuencias de la enfermedad y de las intervenciones. De manera similar, en el estudio canadiense 7 se encontró que el ingreso por urgencia aumentaba el riesgo de reingreso.

\section{Tiempo de estancia}

Se considera que los pacientes con mayor estancia hospitalaria pueden tener una enfermedad más grave o mayor número de comorbilidades y además, están más expuestos a eventos que puedan deteriorar su salud. En el estudio de Jencks et al. 2, el tiempo de estancia mostró mayor influencia en el riesgo de re-hospitalización que otros factores como edad, el sexo, la raza y grado de discapacidad. La mayor estancia hospitalaria también fue identificada como factor predictor de reingresos en los estudios de Zapatero et al. 25 y de van Walraven et al. 36. Nuestras observaciones sobre la estancia y los reingresos coinciden de forma general con lo descrito en la literatura. Podríamos inferir de estos hallazgos que una de las estrategias para evitar los reingresos estaría orientada a disminuir la estancia hospitalaria innecesaria; sin embargo debemos tener en cuenta que otra de las causas de reingreso es el alta temprana 24 .

\section{Costos}

Los reingresos hospitalarios se asocian con mayores costos y, así, pueden afectar el presupuesto destinado a atención en salud. Se sabe que los usuarios más costosos de los hospitales se caracterizan por un patrón de reingresos. En un estudio 37 en 4 estados norteamericanos, en los cuales cerca de $14 \%$ de la población era mayor de 65 años, este mismo grupo de edad causó $62 \%$ del costo de reingresos. La evaluación de los costos 
de los reingresos cobra mayor interés en un país en vías de desarrollo como el nuestro en el cual, el gasto público destinado a la salud es relativamente menor y presenta problemas de distribución ${ }^{38}$. La importancia de los reingresos hospitalarios se refleja también en su impacto sobre los costos desde el punto de vista del asegurador. En este estudio encontramos que los reingresos a los 30 días generan para las compañías aseguradoras cerca del $16 \%$ de los costos por hospitalización. Esa proporción es similar a la estimada para Medicare por Jencks et al. ${ }^{2}$, quienes reportan que los reingresos no planeados representan cerca del $17 \%$ de los costos hospitalarios.

\section{Mortalidad}

Los reingresos pueden al menos en parte reflejar la complejidad de los problemas de salud subyacentes y, por esto mismo, se espera que se asocien a mayor mortalidad. En nuestro estudio, la mortalidad de los reingresos fue claramente mayor que en el grupo que no reingresó $(5,8 \%$ vs. $1,8 \%)$. Sin embargo, no tenemos datos de mortalidad extrahospitalaria. Gorodeski et al. 39 encontraron una relación inversa entre el reingreso y la mortalidad a los 30 días después de la hospitalización en pacientes con insuficiencia cardíaca en 3.857 hospitales. La interpretación de este hallazgo no esperado sería que un hospital que tiene baja mortalidad, tiene mayor proporción de pacientes candidatos a reingresar (sesgo de supervivencia). Por el contrario, Krumholz et al. 40 no encontraron asociación entre reingreso y mortalidad a los 30 días en más de 4 millones de pacientes hospitalizados con infarto agudo de miocardio, insuficiencia cardiaca congestiva o neumonía. Se requieren mayores estudios que permitan aclarar la relación de la mortalidad con los reingresos.

\section{Fortalezas}

Dentro de las fortalezas del presente estudio se destaca el tamaño de la muestra con un gran volumen de población estudiada; la baja pérdida de datos, sin patrón que genere sesgo; la amplia cobertura nacional; la adecuada representación de ambos sexos y grupos de edad, categorías nosológicas; y la inclusión de varios estratos socioeconómicos, ya que cuenta con pacientes de plan obligatorio de salud y pacientes del plan complementario de salud, y la descripción de costos hospitalarios. La base de datos fue diligenciada por médicos y enfermeras profesionales, especialistas en auditoría, lo cual genera una confianza aceptable en la captación y registro de los datos. Teniendo en cuenta que la información de las compañías se alimenta de todos los prestadores a nivel nacional, y que la migración de usuarios entre diferentes aseguradores se puede considerar menor de $1 \%$ en un mes, podemos afirmar además que la base de datos representa bastante bien los reingresos.

\section{Limitaciones}

Falta información adecuada sobre el manejo ambulatorio después del primer egreso registrado, de manera que no se pudo evaluar si el uso de los servicios de urgencias, la oportunidad de acceso a servicios ambulatorios o alguna intervención realizada después del egreso fueron factores relacionados con la probabilidad de reingreso. No realizamos análisis del impacto de la época del año sobre las hospitalizaciones y reingresos. No efectuamos una evaluación de la accesibilidad a servicios de hospitalización, o de la relación de demanda a oferta de servicios hospitalarios. La población del estudio no permite una comparación adecuada con la población colombiana en general $(44,451.147$ personas en el 2008. Departamento Administrativo Nacional de Estadística. http://www.dane.gov.co/index.php/ estadisticas-por-tema/demografia-y-poblacion, accedido el $30 \mathrm{May} / 2017$ ), dado que el grupo de afiliados a la Organización (1.072.863) tiene mayor representación de las clases socioeconómicas más favorecidas, con mayores facilidades de acceso y cobertura a los servicios de salud. No hay en Colombia datos oficiales, ni estudios publicados sobre reingresos hospitalarios, que nos permitan comparar los resultados presentados con los de la población general del país.

Debido a la forma de obtención de la información a partir de una base administrativa hospitalaria, el estudio no contó con el dato de mortalidad extra-hospitalaria, la causa por la cual reingresaron, ni evaluación de pertinencia del reingreso. Tampoco contó con datos sobre variables socioeconómicas, complejidad médica de los casos, calidad de la atención, calidad de vida de los pacientes, dependencia de los pacientes para sus actividades, red de apoyo social, uso de hogar geriátrico u otra institución de manejo extra-hospitalario. No analizamos pacientes que hayan sido hospitalizados más de una vez en el año, por lo que el impacto de este factor sobre los reingresos no se valoró. En la literatura se ha descrito la relación existente entre estos hechos y la frecuencia de reingresos 10,13,17,21,26,41. 


\section{Implicaciones para la investigación y}

\section{para la práctica clínica}

Se requiere de estudios adicionales que evalúen en nuestro medio las contribuciones relativas de dichos factores a los reingresos. Igualmente, es importante identificar y caracterizar los ingresos prevenibles 42,43 . Dada la frecuencia y el costo de los reingresos, es muy importante que nuestros hospitales desarrollen programas tendientes a disminuirlos, teniendo en cuenta los factores que aumentan el riesgo y basados en la mejor evidencia disponible.

\section{Conclusiones}

Uno de cada 10 pacientes que egresan de nuestros hospitales y clínicas reingresan a los 30 días, con mortalidad y estancia mayor, comparadas con el grupo que no reingresa, y consumo del
$16 \%$ de los costos globales de la atención hospitalaria. El mayor número de reingresos correspondió a los pacientes que ingresaron por urgencias, sin embargo, los pacientes remitidos de otras instituciones, tienen el mayor riesgo de reingreso, al igual que los hombres y los mayores de 65 años. La mayor proporción de reingresos ocurre con enfermedades del sistema circulatorio, sin embargo en el análisis multivariado, los grupos de diagnóstico con mayor riesgo de reingreso fueron enfermedades de la sangre y neoplasias. Se requieren estudios prospectivos en nuestro medio que analicen otras variables con potencial impacto en el reingreso tales como: calidad del proceso de atención, programas de seguimiento post-egreso, apoyo social y familiar. Por último, a partir de estos hallazgos se podrá construir modelos para predecir el reingreso de pacientes y desarrollar intervenciones que permitan minimizarlos.

\section{Colaboradores}

A. Caballero coordinó el estudio y contribuyó con la elaboración del protocolo, revisión y análisis de la literatura, introducción, discusión, conclusiones y bibliografía. M. Ibáñez-Pinilla contribuyó con el análisis estadístico de la base de datos, elaboración de tablas, redacción de las secciones materiales y métodos y presentación de resultados. I. C. Suárez-Mendoza y J. R. Acevedo-Peña contribuyeron con la elaboración del protocolo, revisión bibliográfica, análisis de los resultados, discusión y redacción final del trabajo.
Agradecimientos

A la central de auditoría médica por haber proporcionado la base de datos de los egresos hospitalarios. 


\section{Referencias}

1. Epstein AM. Revisiting readmissions - changing the incentives for shared accountability. N Engl J Med 2009; 360:1457-9.

2. Jencks SF, Williams MV, Coleman EA. Rehospitalizations among patients in the Medicare fee-forservice program. N Engl J Med 2009; 360:1418-28.

3. Castro MSM, Carvalho MS, Travassos C. Factors associated with readmission to a general hospital in Brazil. Cad Saúde Pública 2005; 21:1186-200.

4. Evans RL, Hendricks RD, Lawrence KV, Bishop DS. Identifying factors associated with health care use: a hospital-based risk screening index. Soc Sci Med 1988; 27:947-54.

5. Hansen LO, Young RS, Hinami K, Leung A, Williams MV. Interventions to reduce 30-day rehospitalization: a systematic review. Ann Intern Med 2011; 155:520-8.

6. Ortega CG, Barrios JA, Ortega JJG. Tasa de reingresos de un hospital comarcal. Rev Esp Salud Pública 1998; 72:103-10.

7. van Walraven C, Dhalla IA, Bell C, Etchells E, Stiell IG, Zarnke K, et al. Derivation and validation of an index to predict early death or unplanned readmission after discharge from hospital to the community. CMAJ 2010; 182:551-7.

8. McGhan R, Radcliff T, Fish R, Sutherland ER, Welsh C, Make B. Predictors of rehospitalization and death after a severe exacerbation of COPD. Chest 2007; 132:1748-55.

9. Fonarow GC, Abraham WT, Albert NM, Stough WG, Gheorghiade M, Greenberg BH, et al. Factors identified as precipitating hospital admissions for heart failure and clinical outcomes: findings from OPTIMIZE-HF. Arch Intern Med 2008; 168:847-54.

10. Rodriguez-Artalejo F, Guallar-Castillon P, Pascual CR, Otero CM, Montes AO, Garcia AN, et al. Health-related quality of life as a predictor of hospital readmission and death among patients with heart failure. Arch Intern Med 2005; 165:1274-9.

11. Coventry PA, Gemmell I, Todd CJ. Psychosocial risk factors for hospital readmission in COPD patients on early discharge services: a cohort study. BMC Pulm Med 2011; 11:49.

12. Chan FW, Wong FY, Yam CH, Cheung WL, Wong EL, Leung MC, et al. Risk factors of hospitalization and readmission of patients with COPD in Hong Kong population: analysis of hospital admission records. BMC Health Serv Res 2011; 11:186.

13. Vest JR, Gamm LD, Oxford BA, Gonzalez MI, Slawson KM. Determinants of preventable readmissions in the United States: a systematic review. Implement Sci 2010; 5:88.

14. Diz-Lois Palomares M, De la Iglesia Martínez F, Miguel RN, Pellicer Vázquez C, Ramos Polledo V, DizLois Martínez F. Factores predictores de reingreso hospitalario no planificado en pacientes dados de alta de una Unidad de Corta Estancia Médica. An Med Interna (Madrid) 2002; 19:221-5.

15. Superintendencia Nacional de Salud. Circular externa 043 de 2007. http://www.supersalud.gov. co/supersalud/Normatividad/CircularesExternas/HistoricoCircularesExternas (accedido el 14/ Feb/2014)
16. Superintendencia Nacional de Salud. Circular externa 030 de 2006. http://www.supersalud.gov. co/supersalud/Normatividad/CircularesExter nas/HistoricoCircularesExternas (accedido el 14/ Feb/2014).

17. Balla U, Malnick S, Schattner A. Early readmissions to the department of medicine as a screening tool for monitoring quality of care problems. Medicine (Baltimore) 2008; 87:294-300.

18. Bíscaro Valera R, Turrini RNT. Factores relacionados a la readmisión de pacientes en el servicio hospitalario de emergencia. Ciencia y Enfermería 2008; 14:87-95.

19. Alonso Martínez JL, Llorente Díez B, Echegary Agara M, Urbieta Echezarreta M, Gonzáles Arencibia C. Reingreso hospitalario en Medicina Interna. An Med Interna (Madrid) 2001; 18:248-54.

20. Chin MH, Goldman L. Factors contributing to the hospitalization of patients with congestive heart failure. Am J Public Health 1997; 87:643-8.

21. Andersen HE, Schultz-Larsen K, Kreiner S, Forchhammer BH, Eriksen K, Brown A. Can readmission after stroke be prevented? Results of a randomized clinical study: a postdischarge follow-up service for stroke survivors. Stroke 2000; 31:1038-45.

22. Greenblatt DY, Weber SM, O'Connor ES, LoConte NK, Liou J-I, Smith MA. Readmission after colectomy for cancer predicts one-year mortality. Ann Surg 2010; 251:659.

23. Estrada CL, Danielson KK, Drum ML, Lipton RB. Hospitalization subsequent to diagnosis in young patients with diabetes in Chicago, Illinois. Pediatrics 2009; 124:926-34.

24. Halfon P, Eggli Y, van Melle G, Chevalier J, Wasserfallen J-B, Burnand B. Measuring potentially avoidable hospital readmissions. J Clin Epidemiol 2002; 55:573-87.

25. Zapatero A, Barba R, Marco J, Hinojosa J, Plaza S, Losa JE, et al. Predictive model of readmission to internal medicine wards. Eur J Intern Med 2012; 23:451-6.

26. Hasan O, Meltzer DO, Shaykevich SA, Bell CM Kaboli PJ, Auerbach AD, et al. Hospital readmission in general medicine patients: a prediction model. J Gen Intern Med 2010; 25:211-9.

27. Joynt KE, Jha AK. Thirty-day readmissions truth and consequences. N Engl J Med 2012; 366:1366-9.

28. Epstein AM, Jha AK, Orav EJ. The relationship between hospital admission rates and rehospitalizations. N Engl J Med 2011; 365:2287-95.

29. Lagoe RJ, Nanno DS, Luziani ME. Quantitative tools for addressing hospital readmissions. BMC Res Notes 2012; 5:620.

30. Berry JG, Toomey SL, Zaslavsky AM, Jha AK, Nakamura MM, Klein DJ, et al. Pediatric readmission prevalence and variability across hospitals. JAMA 2013; 309:372-80.

31. Navarro E, Mendieta E, Mir R, Caballero B. Readmisiones hospitalarias en recién nacidos egresados de una sala de alojamiento conjunto. Arch Pediatr Urug 2008; 79:86-91. 
32. García-Pérez L, Linertová R, Lorenzo-Riera A Vázquez-Díaz J, Duque-González B, Sarría-Santamera A. Risk factors for hospital readmissions in elderly patients: a systematic review. Q J Med 2011; 104:639-51.

33. Lanièce I, Couturier P, Dramé M, Gavazzi G, Lehman S, Jolly D, et al. Incidence and main factors associated with early unplanned hospital readmission among French medical inpatients aged 75 and over admitted through emergency units. Age Ageing 2008; 37:416-22.

34. Adamuz J, Viasus D, Campreciós-Rodríguez P, Cañavate-Jurado O, Jiménez-Martínez E, Isla P, et al A prospective cohort study of healthcare visits and rehospitalizations after hospital discharge in community-acquired pneumonia. Respirology 2011 16:1119-26.

35. Bahadori K, FitzGerald JM, Levy RD, Fera T, Swiston J. Risk factors and outcomes associated with chronic obstructive pulmonary disease exacerbations requiring hospitalization. Can Respir J 2009; 16:e43-9.

36. van Walraven C, Wong J, Forster AJ. LACE+ index: extension of a validated index to predict early death or urgent readmission after hospital discharge using administrative data. Open Med 2012; 6:e80-e90.
37. Friedman B, Basu J. The rate and cost of hospital readmissions for preventable conditions. Med Care Res Rev 2004; 61:225-40.

38. Preker A, Langenbrunner J. Rich-poor differences in health care financing. In: Dror DM, Preker AS, editors. Social reinsurance: a new approach to sustainable community health financing. Washington DC: World Bank; 2002. p. 21-56.

39. Gorodeski EZ, Starling RC, Blackstone EH. Are all readmissions bad readmissions? N Engl J Med 2010; 363:297-8.

40. Krumholz HM, Lin Z, Keenan PS, Chen J, Ross JS, Drye EE, et al. Relationship between hospital readmission and mortality rates for patients hospitalized with acute myocardial infarction, heart failure, or pneumonia. JAMA 2013; 309:587-93.

41. Peikes D, Chen A, Schore J, Brown R. Effects of care coordination on hospitalization, quality of care, and health care expenditures among Medicare beneficiaries: 15 randomized trials. JAMA 2009; 301:603-18.

42. Joynt KE, Jha AK. A path forward on Medicare readmissions. N Engl J Med 2013; 368:1175-7.

43. van Walraven C, Jennings A, Taljaard M, Dhalla I, English S, Mulpuru S, et al. Incidence of potentially avoidable urgent readmissions and their relation to all-cause urgent readmissions. CMAJ 2011; 183:1067-72. 


\section{Abstract}

Hospital readmissions are common and expensive, and there is little information on the problem in Colombia. The objective was to determine the frequency of 30-day all-cause hospital readmissions and associated factors. This was a retrospective analytical cohort study of 64,969 hospitalizations from January 2008 to January 2009 in 47 Colombian cities. 6,573 hospital readmissions, prevalence: $10.1 \%$ (men 10.9\%, women 9.5\%), $44.7 \%>65$ years of age. Hospital readmissions was associated with higher mortality (5.8\% vs. 1.8\%). There was an increase in the Hospital readmissions rate in patients with diseases of the circulatory system. Hospital readmissions was more likely in hematological diseases and neoplasms. Mean length of stay during the first readmission was 7 days in patients that were readmitted and 4.5 in those without readmission. Greater total cost of hospital readmissions (USA 21,998,275): 15.8\% of the total cost of hospitalizations. Higher prevalence rates in referred patients (18.8\%) and patients from the outpatient clinic (13.7\%). Hospital readmissions is common and is associated with longer length of hospital stay and higher mortality and cost. Increased risk of hospital readmissions in men $>65$ years, patients referred from other institutions, and in hematological diseases and neoplasms.

Hospitalization; Patient Readmission; Health Care Costs

\section{Resumo}

Readmissões hospitalares são comuns e caras; na Colômbia há pouca informação. O objetivo foi determinar a frequência de readmissões hospitalares po qualquer causa aos 30 dias e fatores associados. Estudo analítico, coorte retrospectivo 64.969 hospitalizações entre janeiro de 2008 e janeiro de 2009 em 47 cidades colombianas. Seis mil quinhentos e setenta e três readmissões hospitalares, prevalência: 10,1\% (homens 10,9\%, mulheres 9,5\%), 44,7\% com > 65 anos. Maior mortalidade em readmissões hospitalares $(5,8 \%$ vs. 1,8\%). Aumento do número de readmissões hospitalares no grupo de sistema circulatório. Maior probabilidade de readmissões hospitalares em doenças he matológicas e neoplasias. Tempo médio de internação, durante a primeira readmissão: 7 dias em pacientes que foram readmitidos e 4,5 naqueles sem readmissão. Maior custo total de readmissões hospitalares (USD 21.998.275): 15,8\% do custo total das internações. Maiores prevalências em pacientes encaminhados (18,8\%) e pacientes do ambulatório (13,7\%). Readmis sões hospitalares são comuns, estão associadas com o maior tempo de hospitalização, mortalidade e custo Aumento do risco de readmissões hospitalares em homens com > 65 anos, pacientes encaminhados de outras instituições, doenças hematológicas e neoplasias.

Hospitalização; Readmissão do Paciente;

Custos de Cuidados de Saúde
Recibido el 29/Sep/2014

Versión final presentada el 03/Ago/2015 Aprobado el 18/Feb/2016 